\title{
Hepatoprotective Activity of Yigan Mingmu Oral Liquid against Isoniazid/Rifampicin-Induced Liver Injuries in Rats
}

\author{
Yuxin Chen', Qigui Mo', Baibo Xie ${ }^{2,3}$, Bingxin Ma1, Xinyu Zang2,3, \\ Gao Zhou', Linyou Cheng ${ }^{3}$, James Hua Zhou ${ }^{2,3}{ }^{*}$, Youwei Wang1,4* \\ ${ }^{1}$ Institute of TCM and Natural Products, School of Pharmaceutical Sciences, Wuhan University, Wuhan, China \\ ${ }^{2}$ Beijing Hebabiz Biotechnology Co. Ltd., Beijing, China \\ ${ }^{3}$ National and Region joint Engineering Center for Anticancer Drug Development, Guangxi Hebabiz Pharmaceutical Co. Ltd., \\ Qinzhou, China \\ ${ }^{4}$ MOE Key Laboratory of Combinatorial Biosynthesis and Drug Discovery, Wuhan University, Wuhan, China \\ Email: *wyw@whu.edu.cn, *drjge@163.com
}

How to cite this paper: Chen, Y.X., Mo, Q.G., Xie, B.B., Ma, B.X., Zang, X.Y., Zhou, G., Cheng, L.Y., Zhou, J.H. and Wang, Y.W. (2018) Hepatoprotective Activity of Yigan Mingmu Oral Liquid against Isoniazid/Rifampicin-Induced Liver Injuries in Rats. Chinese Medicine, 9, 165-178. https://doi.org/10.4236/cm.2018.94010

Received: May 2, 2018

Accepted: December 2, 2018

Published: December 5, 2018

Copyright $(0) 2018$ by authors and Scientific Research Publishing Inc. This work is licensed under the Creative Commons Attribution International License (CC BY 4.0).

http://creativecommons.org/licenses/by/4.0/

\section{(c) (i) Open Access}

\begin{abstract}
Background: To explore the hepatoprotective effect of Yigan mingmu oral liquid (YGMM) on isoniazid-rifampicin induced liver injury in rats. Methods: Total 38 SD rats were randomly divided into 6 groups including control group, model group, silymarin positive control group, and three YGMM treatment groups. Model group was administered intragastrically with INH (100 mg/kg) and RIF (100 mg/kg) for 14 days. Silymarin group and YGMM treatment groups were administered intragastrically with silymarin (100 $\mathrm{mg} / \mathrm{kg})$ and different doses of YGMM $(1,2.5,5 \mathrm{mg} / \mathrm{kg}) 2$ hours before INH and RIF administration from day 4 to day 14. Results: Rats were sacrificed 16 hours after the last day treatment to determine the activities of serum alanine transaminase (ALT), aspartate transaminase (AST) and alkaline phosphatase (ALP), as well as total bilirubin (TB) content. Oxidative stress was evaluated by measuring total superoxide dismutase (T-SOD) and malondialdehyde (MDA) levels. Histopathological changes in liver tissues were observed under an optical microscope by using hematoxylin and eosin staining. The mice in model groups showed significantly $(p<0.05)$ increased levels in AST, ALT, ALP, TB and MDA compared to their control groups; and showed significantly $(p<0.05)$ decreased level in T-SOD. These changes were significantly $(p<0.05)$ reversed by the YGMM treatments in a dose-dependent manner. Hepatic pathological changes were attenuated or even reversed by silymarin or YGMM treatments. Conclusions: YGMM has a good hepatoprotective activity on isoniazid-rifampicin induced liver injuries in rats.
\end{abstract}




\section{Keywords}

Herbal Prescription, Hepatoprotective Activity, Isoniazid, Rifampicin, Oxidative Stress

\section{Background}

Isoniazid (INH) and rifampicin (RIF) are the most common medications prescribed for tuberculosis treatment, which are the first line drugs used for tuberculosis chemotherapy but also are the most common agents that induce liver disease [1]. Combined medication of INH and RIF can greatly increase the incidence of liver injury and disease severity; severe cases may lead to liver necrosis and even liver failure [2] [3]. Metabolic site of INH and RIF is the liver, which increases the possibility of liver injury. Liver damage caused by anti-tuberculosis drugs belongs to a drug complication that commonly happened during the anti-tuberculosis treatment [4], as well as an important factor affecting the efficacy of anti-tuberculosis treatment and the prognosis of patients. So far, little is known about the cellular and biochemical mechanisms of INH- or RIF-induced hepatotoxicity, but oxidative stress is considered as one of the mechanisms underlying INH + RIF-induced hepatic injury [5].

As the basis of the classic prescription Siwu Tang [6] and the "Gugen Tang" created by Qing Dynasty Shiduo Chen, Yigan mingmu oral liquid (YGMM) was extended by addition of wolfberry (Lycium barbarum or Lycium chinense) fruit and cassia seed. The medicinal preparation contains 12 traditional Chinese medicines (TCM). Rehmannia glutinosa is taken to enrich the blood, nourish Yin and invigorate the kidney in its traditional use, and has been proved as an effective drug for treatment of diabetes [7] and benefit for cardiovascular system [8]. Angelica sinensis is mainly used for the treatment of women's reproductive problems, such as dysmenorrhea, amenorrhoea, menopause, among others [9]. Modern medical researches demonstrate that it has antioxidant, anti-tumor activity, immunoregulatory activity, neuroprotective effect, etc. [10]. White peony root, processed product from the root of Paeonia lactiflora Pall, is traditionally used for alleviating pain, tonifying blood and regulating menstruation. Anti-depressant effect [11], anti-inflammatory and immunomodulatory effects [12] of Paeonia lactiflora Pall have been reported recently. Polygonatum odoratum, Ophiopogon japonicas, and wolfberry fruit have been used for centuries in China as a nourish $Q i$ and $Y i n$, and invigorate the liver and kidney medicine. Polygonatum odoratum has been proved having antioxidative effect [13]; Ophiopogon japonicus has antithrombotic activities [14], anti-inflammatory activities [15], antioxidant and immunoregulatory activity [16]. Multiple pharmacological activities of wolfberry fruit have been reported, such as hepatotoxicity [17], hypoglycemic and hypolipidemic effects [18], antioxidant and anti-aging effects [19], and so on. Chrysanthemum flower and cassia seed are traditionally used 
for clearing liver and improving eyesight. The hepatoprotective effect of Chrysanthemum flower has been proved by Jeong et al. [20]. Chrysanthemum flower and cassia seed are demonstrated to have antimicrobial activity respectively by scientific research [21] [22]. All the herbs mentioned above are the main constitution of the YGMM, along with Ligusticum chuanxiong, Anemone altaica, Citrus reticulate, and Bupleurum spp., which are the complementary constitution of the YGMM, respectively used for promoting blood and Qi circulation; resuscitation with aromatics; regulating Qi flow and strengthening spleen; and promoting Qi flow and soothing liver in TCM.

The herbal medicine of YGMM was registered and approved by CFDA (Approval No. B20050056) for treating soreness and weakness of waist and knees; dizziness; memory loss; and fatigue. We speculate that YGMM may have the effects to protect liver from injuries, as well as treating liver related diseases based on the traditional usage of prescription ingredients. However, there is a lack of data to elaborate its hepatoprotective activity. Therefore, our objectives in the current study are to observe the effects of YGMM on biochemical parameters and pathological changes of liver caused by combination of INH and RIF and to investigate the ameliorating effect of YGMM on drug-induced liver injury and expand its clinical application.

\section{Materials and Methods}

\subsection{Chemicals}

YGMM was provided by Guangxi Hebabiz Pharmaceutical Co., Ltd. (Lot No. 130418). RIF was purchased from Fourth Pharmaceutical Co., Ltd. of the Zhong Lian Group (Wuhan, China; Lot No. 131002). INH was purchased from Shantou Jinshi General Pharmaceutical Factory (Shantou, China; Lot No. 130502). Silymarin was purchased from MADAUS Gmbh, Germany (Lot No. B1102662). Aspartate amino transferase (AST), alanine amino transferase (ALT), alkaline phosphatase (ALP), total bilirubin (TB), superoxide dismutase (SOD) and malondialdehyde (MDA) assay kits were purchased from the Institute of Biological Engineering of Nanjing Jiancheng (Nanjing, China), lot number is $20140327,20140327,20140402,20140329,20140219,20140216$, respectively.

\subsection{Test Animals}

A total of 38 Sprague-Dawley rats (male:females $=1: 1$; each animal weighing 200 $\mathrm{g} \pm 20 \mathrm{~g}$ ) were used for hepatic damage studies. The animals were purchased from the Laboratory Animal Center of Wuhan University (Wuhan, China). The rats were grouped and housed under $12 \mathrm{~h} / 12 \mathrm{~h}$ light-dark cycle with food and water provided ad libitum at $25^{\circ} \mathrm{C} \pm 2{ }^{\circ} \mathrm{C}$ and $30 \%$ to $60 \%$ relative humidity. The experimental protocol was sanctioned by the Institutional Animal Care and Use Committee (IACUC) at Center for Animal Experiment, Wuhan University (Wuhan, China). 


\subsection{INH + RIF-Induced Liver Injury}

After an acclimatization period of 3 days, 38 rats were randomly divided into 6 groups. Each group contains 6 rats except model group contains 8 rats.

We used body surface area algorithm that was proposed by Shuyun $\mathrm{Xu}$ in 1982 [23] to calculate the doses of YGMM. The prescribed oral dose of YGMM is $10 \mathrm{ml}$ once, 3 times a day. We consider that a $60 \mathrm{~kg}$ person has a clinical dose of $30 \mathrm{ml} / 60 \mathrm{~kg}$ per day, which is converted into a $200 \mathrm{~g}$ rat dose.

$$
\text { Rat dose }=0.5 \mathrm{ml} / \mathrm{kg} \times 60 \mathrm{~kg} \times 0.018 / 0.2 \mathrm{~kg}=2.7 \mathrm{ml} / \mathrm{kg}
$$

( 0.018 is a body surface area factor)

Therefore, we chose $2.5 \mathrm{ml} / \mathrm{kg}$ as our middle dose, then $5 \mathrm{ml} / \mathrm{kg}$ as high dose and $1 \mathrm{ml} / \mathrm{kg}$ as low dose for rats.

The design of the animal work refers to Pal et al. [24] and Dong et al. [25] and slightly modified.

Group A (control group) received a daily dose of $0.3 \%$ CMC-Na $(10 \mathrm{~mL} / \mathrm{kg}$ bodyweight, i.g.) for $14 \mathrm{~d}$.

Group B (INH + RIF-induced liver injury model group) received a daily dose of INH (100 mg/kg, i.g.) + RIF (100 mg/kg, i.g.) for $14 \mathrm{~d}$.

Group C (positive control group) was treated with the standard drug silymarin $(100 \mathrm{mg} / \mathrm{kg}$, i.g.) daily for $11 \mathrm{~d}$. For the first 3 days, the group only received INH $(100 \mathrm{mg} / \mathrm{kg}$, i.g. $)+$ RIF $(100 \mathrm{mg} / \mathrm{kg}$, i.g.). From day 4 onward, the group was intragastrically administered INH + RIF $2 \mathrm{~h}$ after the administration of silymarin.

Groups D, E, and F (low-, medium-, and high-dose YGMM treatment groups) were treated with three different doses of YGMM $(1,2.5$, and $5 \mathrm{ml} / \mathrm{kg}$, respectively, i.g.) for 11 d. For the first 3 days, these groups only received INH (100 $\mathrm{mg} / \mathrm{kg}$, i.g.) + RIF (100 mg/kg, i.g.). From day 4 onward, these groups were intragastrically treated with INH + RIF $2 \mathrm{~h}$ after the administration of different doses of YGMM.

\subsection{Liver Index and Kidney Index Determination}

At the end of the experiments, the rats were starved overnight and sacrificed the next day by cervical dislocation. The body weights of rats were recorded before sacrificed and the weights of livers and kidneys were recorded immediately after the rats were sacrificed. Liver index $=$ liver weight/body weight $\times 100 \%$; Kidney index $=$ kidney weight/body weight $\times 100 \%$.

\subsection{Biochemical Determinations in Serum}

Blood samples were collected from the heart artery and allowed to coagulate. The serum was isolated from blood by centrifuging at $3000 \mathrm{rpm}$ for $10 \mathrm{~min}$ and conserved at $-20^{\circ} \mathrm{C}$ for subsequent analysis. The activities of AST, ALT, ALP and the concentration of $\mathrm{TB}$ in the serum were determined in accordance with the protocols provided by the manufacturers of the kits. The activities of AST, ALT, and ALP were expressed in U per L, whereas the level of TB was expressed 
in $\mathrm{mg}$ per $\mathrm{dL}$.

\subsection{Measurement of Antioxidant Enzymes and MDA Levels in Liver and Kidney}

Liver and kidney samples were dissected and rinsed in ice-cold saline to remove the blood. The liver and kidney homogenates $(10 \%, w / v)$ were prepared in cold $50 \mathrm{mM}$ potassium phosphate buffer ( $\mathrm{pH}$ 7.4). The resulting suspension was centrifuged at $5000 \mathrm{rpm}$ for $10 \mathrm{~min}$ at $4^{\circ} \mathrm{C}$. The total protein contents were determined by Bradford method. The protein concentration was calculated from the calibration curve: $\mathrm{C}=190.38 \mathrm{~A}-12.40$ (C: concentration of protein; A: absorbance at $595 \mathrm{~nm}$; and $\mathrm{R}^{2}=0.9992$ ). The activities of both SOD and CAT were estimated using commercially available kits. The results were expressed in $\mathrm{U}$ per $\mathrm{mg}$ protein. The contents of GSH and MDA were expressed in mg per g protein and nmol per mg protein, respectively, based on the protocol provided by the manufacturers of the kits.

\subsection{Histopathological Examination of Liver Tissue}

A small piece of the liver tissue was cut from liver and immediately transferred into $10 \%$ formalin to fix for $24 \mathrm{~h}$ and was embedded in paraffin wax. Then cut the blocks into $5 \mu \mathrm{m}$-thick sections in a rotary microtome (RM2125, Leica, US). The sections were stained with hematoxylin and eosin. Each slice was microscopically (IX51, Olympus, Japan) examined to observe the distribution of histopathological changes.

\subsection{Statistical Analysis}

All data were presented as mean \pm S.E.M. Statistical analysis was performed using IBM SPSS 20.0 for Windows. One-way analysis of variance (ANOVA) followed by LSD test was conducted for multiple comparisons. Statistical significance was determined at $p<0.05, p<0.01$, and $p<0.001$ levels.

\section{Results and Discussion}

\subsection{Liver Index and Kidney Index Determination}

Figure 1 shows the liver index and kidney index of the rats after 14 days' treatment. From the changes in liver index (Figure 1(A)), we can see that the liver index of rats in the INH + RIF model group decreased significantly $(p<0.05)$ compared to the control group. The silymarin group and three YGMM treatment groups (YGMMs) had no significant effect on increasing liver index compared to the model group, but there is a trend showing that liver index can be slightly increased by silymarin and low-dose YGMM treatment compared to the model group. The same observation that INH + RIF co-administration induces the decrease of relative liver weight was reported by Pal et al. [24]. From the changes in kidney index (Figure 1(B)), we can see that the kidney index of rats in the INH + RIF model group increased significantly $(p<0.05)$ compared with 

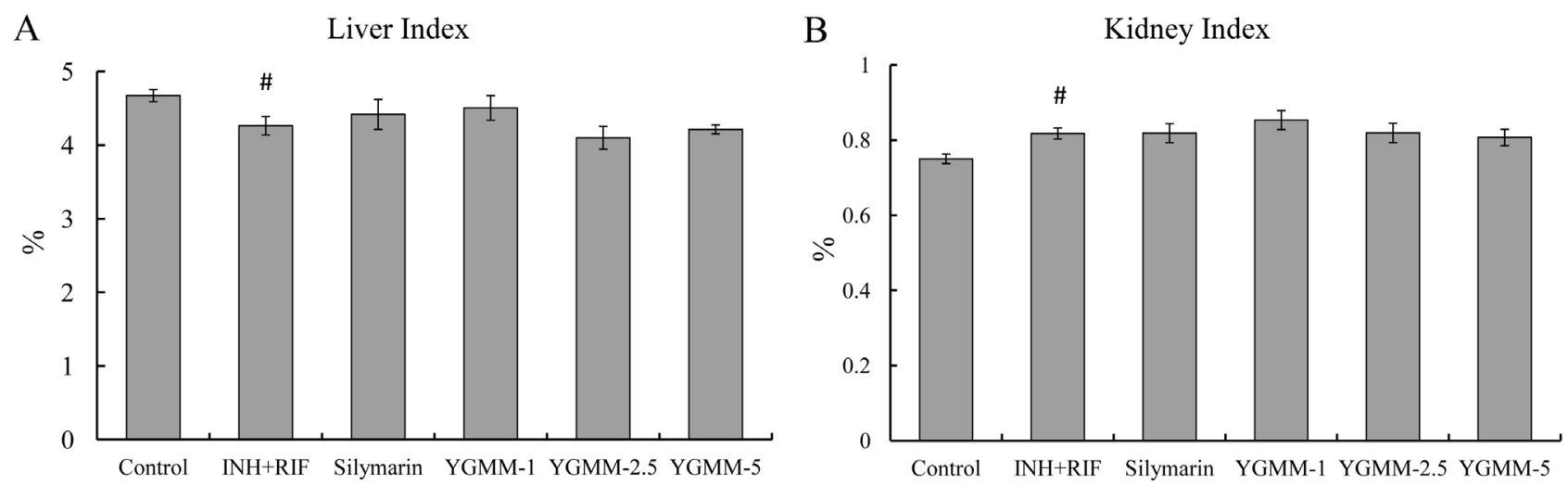

Figure 1. Effects of YGMM on the (A) liver index and (B) kidney index of INH + RIF-treated rats receiving YGMM or silymarin supplementation. ${ }^{*} p<0.05$ indicate significant differences between model group and control group; there are no significant differences between silymarin or YGMMs and model group.

the control group.

The silymarin group and YGMMs had no significant effect on decreasing kidney index compared to model group. With YGMM dose increased, the kidney index gradually decreased, which can be considered that YGMM has an effect on reversing kidney index to control level in a dose-dependent manner. There is no report on that INH and RIF co-administration can induce the increase of relative kidney weight. This is the first paper to report that INH and RIF co-administration has the effect on inducing kidney hypertrophy statistically.

\subsection{Biochemical Determinations in Serum}

The co-administration of INH and RIF for $14 \mathrm{~d}$ induced liver injury, as indicated by a marked increase of serum ALT, AST, ALP, and TB levels (Figures 2(A)-(D)). The corresponding indicators of the rats treated with silymarin and YGMM were lower than that in the INH + RIF-intoxicated groups.

YGMM pretreatment at $5 \mathrm{ml} / \mathrm{kg}$ significantly $(p<0.05, p<0.001, p<0.05, p<$ 0.01 , respectively) prevented the elevation of these marker enzymes, and these effects was almost comparable with those observed for corresponding silymarin-treated groups. As we know, there are many common animal model of acute liver injury, such as modeling with carbon tetrachloride, modeling with alcohol, and modeling with some immune agents. After the introduction of INH in 1969, the problem of INH-induced hepatitis remained. Previous studies postulated that the hepatotoxicity of INH was initiated by cytochrome P450-mediated metabolism of INH to acetylhydrazine and hydrazine [26]. RIF is a powerful inducer of mixed function oxidase in man and rats and contributes to the hepatotoxicity of INH by enhancing the production of toxic metabolites [27]. The combination of INH and RIF as antitubercular drugs has a long history stated that INH and RIF administered in combination resulted in more severe effects than when INH was administered alone [28]. Moreover, elevated transaminase levels were observed in clinical cases administered with INH and RIF [29]. 

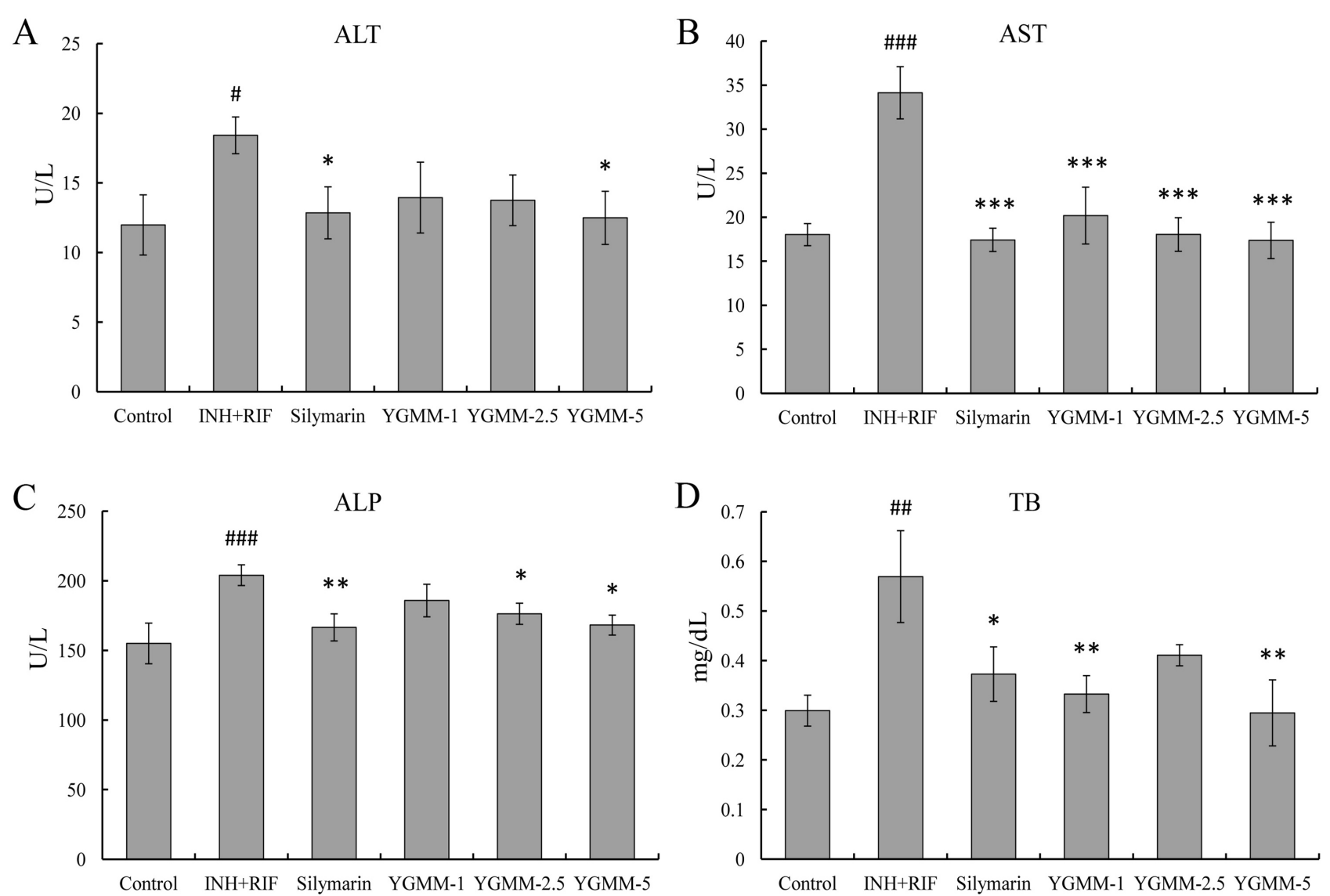

Figure 2. Effects of YGMM on the (A) ALT, (B) AST, (C) ALP activities, and (D) the level of TB in the serum of INH + RIF-treated rats receiving YGMM or silymarin supplementation. ${ }^{\#} p<0.05,{ }^{\# \#} p<0.01$, and ${ }^{\# \#} p<0.001$ indicate significant differences between model group and control group; ${ }^{\star} p<0.05,{ }^{* *} p<0.01$, and ${ }^{\star * *} p<0.001$ indicate significant differences between silymarin or YGMMs and model group.

\subsection{Levels of Antioxidant Enzyme and MDA in Liver and Kidney}

The INH + RIF-induced liver injury group exhibited significant $(p<0.05)$ reduction in liver SOD (Figure 3(A)) and CAT (Figure 3(B)) activities, produced a significant consumption of liver GSH (Figure 3(C)) content, and showed a remarkable promotion of liver MDA (Figure 3(D)) content compared with the control group. The hepatoprotective effect of YGMM at the highest dose significantly $(p<0.05)$ elevated the reduction in SOD, CAT, and GSH concentrations and reduced the increase in MDA level compared to the model group. The highest dose YGMM showed the competitive effect on reversing the SOD, CAT, and GSH levels and better effect on decreasing MDA level compared to silymarin's treatment.

The INH + RIF administrated group exhibited significant $(p<0.05)$ reduction in kidney SOD (Figure 4(A)) and CAT (Figure 4(B)) activities, produced a significant consumption of kidney GSH (Figure $4(\mathrm{C})$ ) content, and showed a remarkable promotion of kidney MDA (Figure 4(D)). An unexpected protective effect was observed in kidney tissues. The highest dose of YGMM significantly ( $p<$ 0.05 ) maintained the normal levels of CAT, GSH, and MDA (Figures 4(B)-(D)), 

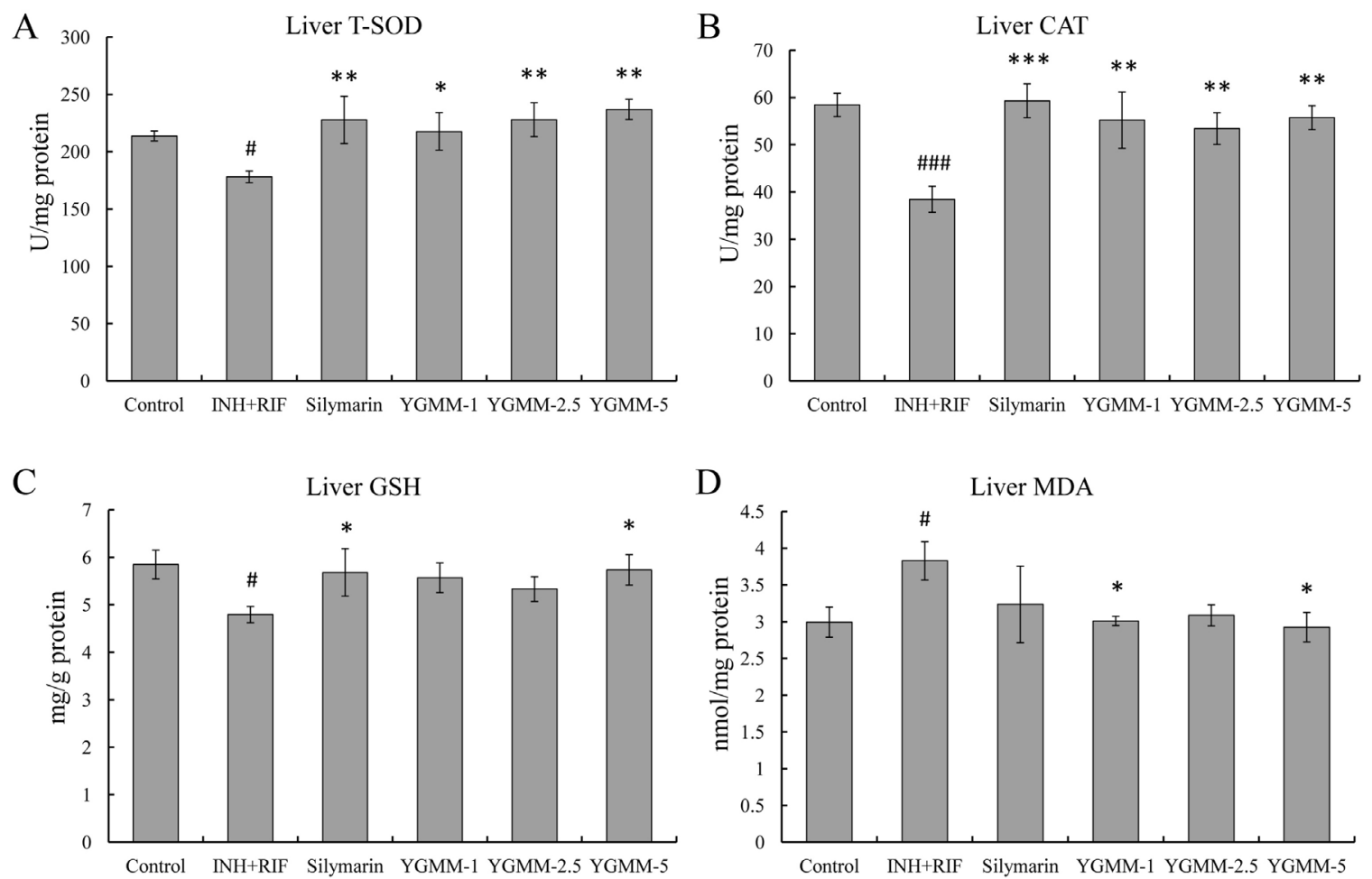

Figure 3. The (A) T-SOD, (B) CAT, (C) GSH activities, and (D) the levels of MDA in the liver of INH + RIF-treated rats receiving YGMM or silymarin supplementation. ${ }^{*} p<0.05$ and ${ }^{\# \# \#} p<0.001$ indicate significant differences between model group and control group; ${ }^{\star} p<0.05,{ }^{\star *} p<0.01$, and ${ }^{\star * *} p<0.001$ indicate significant differences between silymarin or YGMMs and model group.
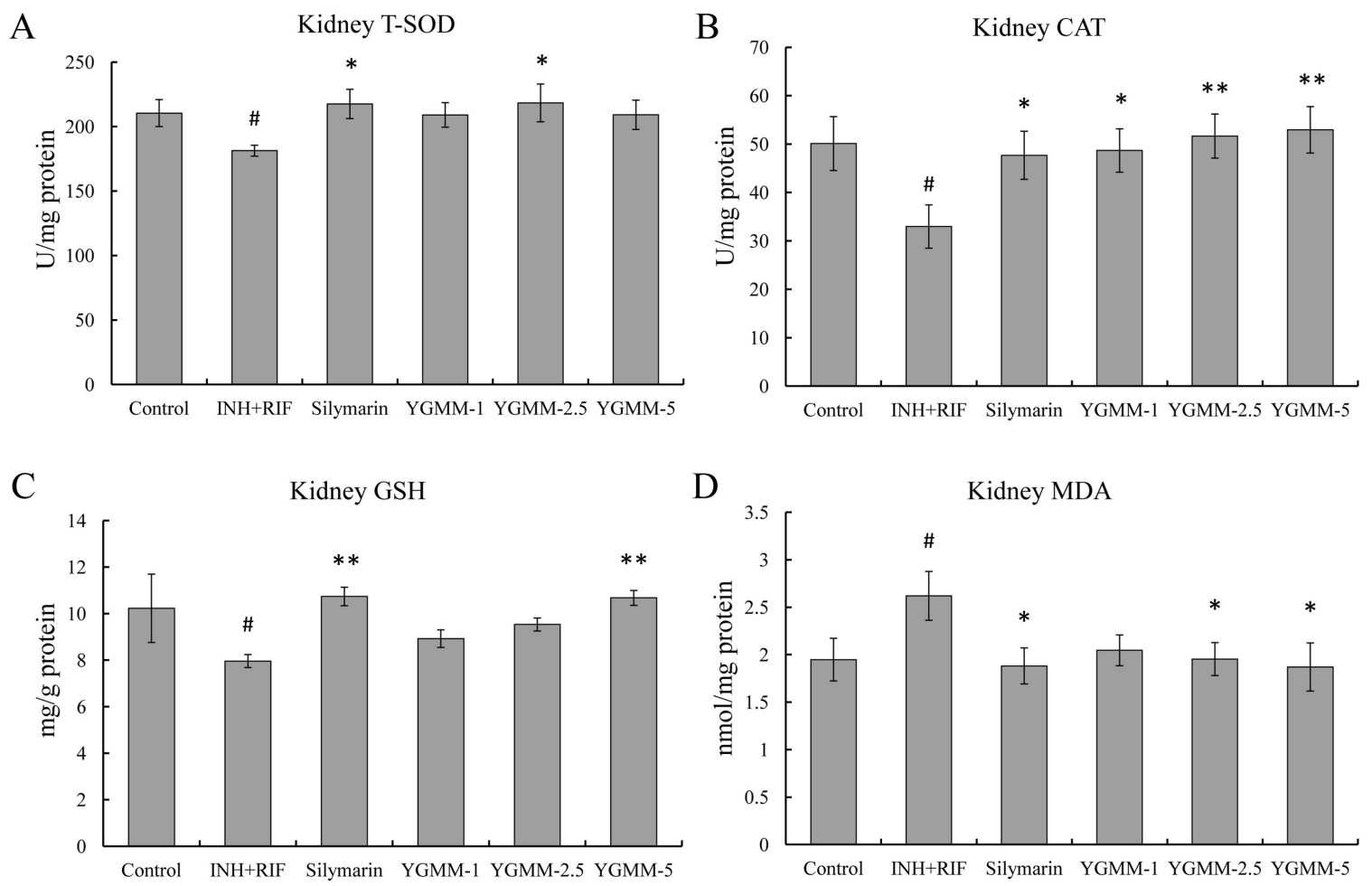

Figure 4. The (A) T-SOD, (B) CAT, (C) GSH activities, and (D) the levels of MDA in the kidney of INH + RIF-treated rats receiving YGMM or silymarin supplementation. ${ }^{*} p<0.05$ indicate significant differences between model group and control group; ${ }^{*} p<0.05$ and ${ }^{* *} p<0.01$ indicate significant differences between silymarin or YGMMs and model group. 
whereas SOD activity was maintained at near normalcy when the animals were treated with $2.5 \mathrm{ml} / \mathrm{kg}$ YGMM (Figure 4(A)). Overall, YGMM exhibited a strong effect on restoring antioxidant capacity of kidney against the detrimental effect caused by INH + RIF combination in a dose-dependent manner.

To prevent the damage caused by oxygen-free radicals, tissues develop an antioxidant defense system, which includes non-enzymatic antioxidants (e.g., glutathione, uric acid, bilirubin, and vitamins $\mathrm{C}$ and $\mathrm{E}$ ) and enzymatic antioxidants, such as SOD, CAT, and glutathione peroxidase. SOD, CAT, and glutathione peroxidase have important physiological functions. The activities of these compounds are easily reduced by lipid peroxide or ROS [30]. SOD converts $\mathrm{O}_{2}$ into $\mathrm{H}_{2} \mathrm{O}_{2}$, and CAT metabolizes $\mathrm{H}_{2} \mathrm{O}_{2}$ to $\mathrm{H}_{2} \mathrm{O}$ and $\mathrm{O}_{2}$. GSH acts as antioxidant in conjunction with various enzymatic processes that reduce hydrogen peroxide and hydroperoxide by oxidizing GSH to glutathione disulfide and other mixed disulfides [31]. The non-enzymatic lipid peroxidation of living organisms is a natural, continually occurring process. However, the levels of non-enzymatic lipid peroxidation can dramatically increase under oxidative stress conditions when ROS and radical production increase [32]. Previous studies focused on the toxic effects of lipid-derived carbonyl compounds. For example, compounds such as 4-hydroxy-2(E)-nonenal and MDA have been found to trigger apoptosis, cause mutations, and act as respiratory toxins when exogenously administered to animal cells [33].

The mechanism of INH + RIF-induced hepatotoxicity remains unknown. Sarich et al. [34] have reported that both acetylhydrazine and hydrazine exist during active INH-induced hepatotoxicity in rabbits, thereby indicating that hydrazine is most likely involved in the mechanism of hepatic necrosis. Shih et al. [35] observed that RIF increases INH toxicity, which was consistent with the results obtained from human studies. Oxidative stress may be one of the mechanisms by which INH + RIF causes liver damage. Chowdhury et al. [36] detected pronounced oxidative stress in the mitochondria of INH + RIF-treated mice liver cells. Such oxidative stress induced the increase in non-protein and protein thiol oxidation. Santhosh et al. [37] proved that INH + RIF-induced alterations in protein metabolism and hepatic antioxidant defense system were normalized by chitosan co-administration, thereby indicating the possible cytoprotective role of chitosan. $\mathrm{N}$-acetylcysteine was confirmed to resist oxidative hepatic injury caused by INH + RIF [38]. If oxidative stress was the main mechanism underlying INH + RIF toxicity, we are for the first time to confirm that YGMM can reduce oxidative stress caused by INH + RIF. These protection effects of YGMM for liver and kidney we observed may be attributed, at least partly, to the strong antioxidant activity of YGMM.

\subsection{Histopathological Examination of Liver Tissue}

For the histopathological observations of the liver sections of INH + RIF-induced liver injury rats, Group A (Figure 5(A)) showed normal lobular architecture and 

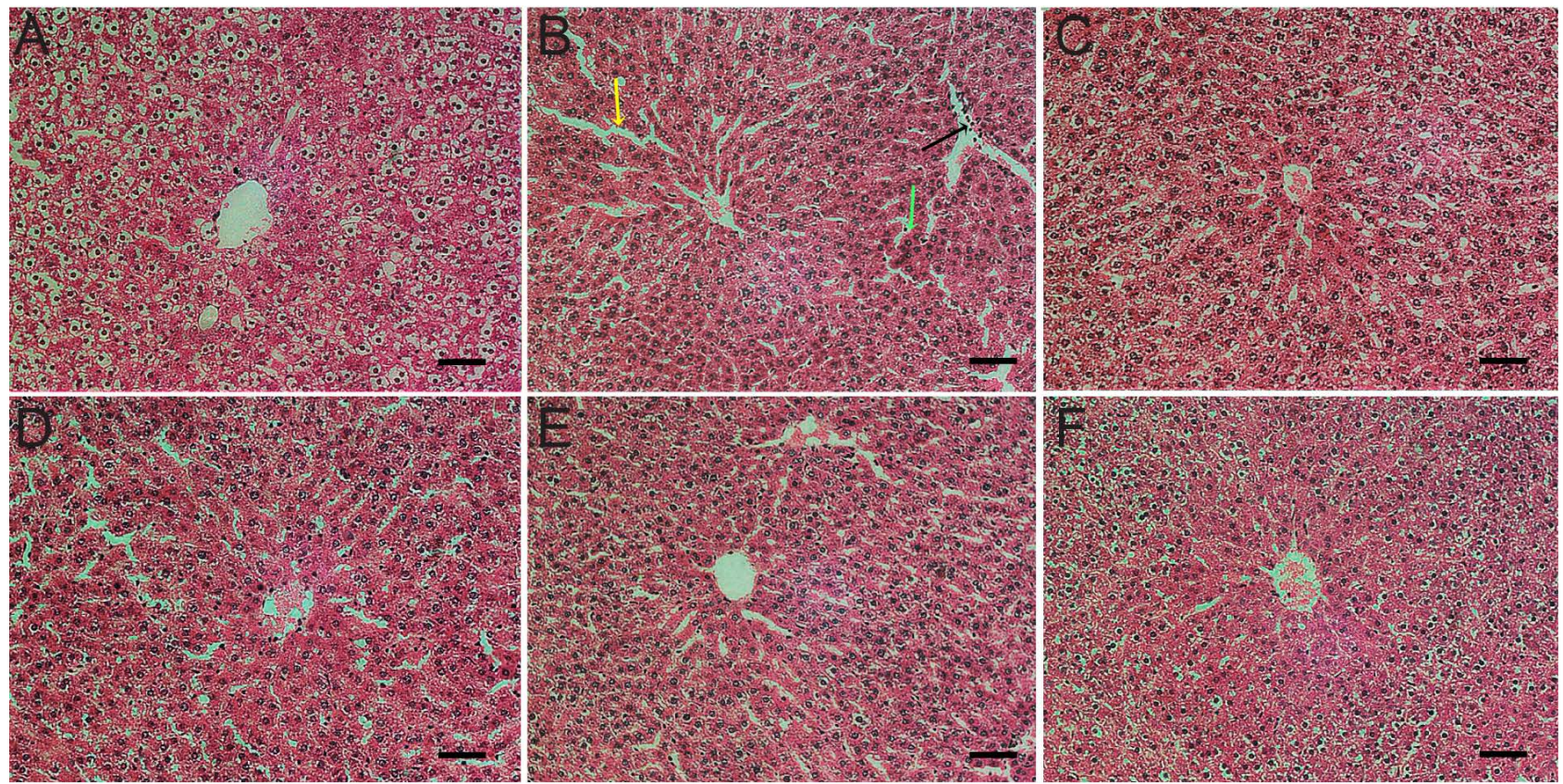

Figure 5. Effects of YGMM on the liver histological structure of rats with INH + RIF-induced liver injury (hematoxylin-eosin staining, 200×). The treatments were as follows: (A) Control; (B) INH (100 mg/kg) + RIF (100 mg/kg); (C) INH + RIF + silymarin $(100 \mathrm{mg} / \mathrm{kg}) ;(\mathrm{D}) \mathrm{INH}+\mathrm{RIF}+\mathrm{YGMM}(1 \mathrm{ml} / \mathrm{kg}) ;(\mathrm{E}) \mathrm{INH}+\mathrm{RIF}+\mathrm{YGMM}(2.5 \mathrm{ml} / \mathrm{kg}) ;$ and (F) INH + RIF + YGMM (5 ml/kg). The yellow arrow indicates the disordered lobular structure, the green arrow indicates patchy sinusoidal congestion, and the black arrow indicates mononuclear inflammatory cells infiltration, scale bar: $50 \mu \mathrm{m}$.

liver plates. No significant sinusoidal dilation was observed. Expansion and fibrosis of periportal and interface inflammation were not observed. Liver cells showed no significant necrosis, regeneration, and degeneration. Dense and compactness nuclear was observed. In group B (model group; Figure 5(B)), lobular structure was observed as slightly disordered (yellow arrow). Patchy sinusoidal congestion (green arrow) and mononuclear inflammatory cell infiltration (black arrow) were observed in the portal area. Liver cells of groups C, D, E, and F showed the abovementioned characteristics, but some improvements were evident compared with the model group. Among the groups, groups $\mathrm{C}$ and $\mathrm{F}$ showed the best treatment effects. From Figures 5(D)-(F), we can see that nuclear restore dense and compactness gradually, which can be considered that the effects of YGMM on reversing INH+RIF inducing liver injury were demonstrated in a dose-dependent manner.

\section{Conclusion}

In this paper, YGMM was evaluated for hepatoprotective activity against INH/RIF-induced liver injuries in rats. YGMM exhibited strong hepatoprotective activity, thereby confirming the traditional use of YGMM as an herbal prescription. The maximum protection against hepatic damage was achieved with the highest tested dose of YGMM (5 ml/kg). The possible YGMM mechanisms of action could include its ability to protect the liver against cellular oxidative damage and to maintain the intracellular level of antioxidant enzymes. Thus, 
YGMM can be used as a potential hepatoprotective agent or health care product for those people who are under risk of liver injuries while accepting INH and RIF treatment against tuberculosis.

\section{Acknowledgements}

Guangxi Hebabiz Pharmaceutical Co., Ltd. (P.R. China) provided Yigan mingmu oral liquid (YGMM) for this work.

\section{Conflicts of Interest}

The authors declared that there are no conflicts of interest.

\section{Consent for Publication}

All of authors consent to publication of this study in Journal of Chinese Medicine.

\section{References}

[1] Yee, D., Valiquette, C., Pelletier, M., Parisien, I., Rocher, I. and Menzies, D. (2003) Incidence of Serious Side Effects from First-Line Antituberculosis Drugs among Patients Treated for Active Tuberculosis. American Journal of Respiratory and Critical Care Medicine, 167, 1472-1477. https://doi.org/10.1164/rccm.200206-626OC

[2] Costiniuk, C.T., Gosnell, B.I., Manzini, T.C., Du Plessis, C.N. and Moosa, M.Y. (2015) Tuberculous Drug-Induced Liver Injury and Treatment Re-Challenge in Human Immunodeficiency Virus Co-Infection. Journal of Global Infectious Diseases, 7, 151-156. https://doi.org/10.4103/0974-777X.170499

[3] Wang, C., Fan, R.Q., Zhang, Y.X., Nie, H. and Li, K. (2016) Naringenin Protects against Isoniazid- and Rifampicin-Induced Apoptosis in Hepatic Injury. World Journal of Gastroenterology, 22, 9775-9783. https://doi.org/10.3748/wjg.v22.i44.9775

[4] Yew, W. W. (2002) Clinically Significant Interactions with Drugs Used in the Treatment of Tuberculosis. Drug Safety, 25, 111-133. https://doi.org/10.2165/00002018-200225020-00005

[5] Baniasadi, S., Eftekhari, P., Tabarsi, P., Fahimi, F., Raoufy, M.R., Masjedi, M.R. and Velayati, A.A. (2010) Protective Effect of N-Acetylcysteine on Antituberculosis Drug-Induced Hepatotoxicity. European Journal of Gastroenterology \& Hepatology, 22, 1235-1238. https://doi.org/10.1097/MEG.0b013e32833aa11b

[6] Su, S.L., Cui, W.X., Zhou, W., Duan, J.A., Shang, E.X. and Tang, Y.P. (2013) Chemical Fingerprinting and Quantitative Constituent Analysis of Siwu Decoction Categorized Formulae by UPLC-QTOF/MS/MS and HPLC-DAD. Chinese Medicine, 8 , 5. https://doi.org/10.1186/1749-8546-8-5

[7] Zhu, H.F., Wang, Y., Liu, Z.Q., Wang, J.H., Wan, D., Feng, S., et al. (2016) Antidiabetic and Antioxidant Effects of Catalpol Extracted from Rehmannia glutinosa (Di Huang) on Rat Diabetes Induced by Streptozotocin and High-Fat, High-Sugar Feed. Chinese Medicine, 11, 25. https://doi.org/10.1186/s13020-016-0096-7

[8] Zhou, J., Xu, G., Yan, J., Li, K., Bai, Z., Cheng, W. and Huang, K. (2015) Rehmannia glutinosa (Gaertn.) DC. Polysaccharide Ameliorates Hyperglycemia, Hyperlipemia and Vascular Inflammation in Streptozotocin-Induced Diabetic Mice. Journal of Ethnopharmacology, 164, 229-238. https://doi.org/10.1016/j.jep.2015.02.026 
[9] Hook, I.L.I. (2014) Danggui to Angelica sinensis Root: Are Potential Benefits to European Women Lost in Translation? Journal of Ethnopharmacology, 152, 1-13. https://doi.org/10.1016/j.jep.2013.12.018

[10] Chen, X.P., Li, W., Xiao, X.F., Zhang, L.L. and Liu, C.X. (2013) Phytochemical and Pharmacological Studies on Radix Angelica sinensis. Chinese Journal of Natural Medicines, 11, 577-587. https://doi.org/10.1016/S1875-5364(13)60067-9

[11] Yu, X.H., Song, T., Hou, X.L., Sui, Y., Li, Y.L., Hu, D., et al. (2017) Anti-Depressant Effect of Paeonia lactiflora Pall Extract in Rats. Tropical Journal of Pharmaceutical Research, 16, 577-580. https://doi.org/10.4314/tjpr.v16i3.11

[12] He, D.Y. and Dai, S.M. (2011) Anti-Inflammatory and Immunomodulatory Effects of Paeonia lactiflora Pall., a Traditional Chinese Herbal Medicine. Frontiers in Pharmacology, 2, 10. https://doi.org/10.3389/fphar.2011.00010

[13] Yang, H.J. and Zhang, X.H. (2017) Polysaccharides from Polygonatum odoratum Strengthen Antioxidant Defense System and Attenuate Lipid Peroxidation against Exhaustive Exercise-Induced Oxidative Stress in Mice. Tropical Journal of Pharmaceutical Research, 16, 795-801. https://doi.org/10.4314/tjpr.v16i4.8

[14] Kou, J., Tian, Y., Tang, Y., Yan, J. and Yu, B. (2006) Antithrombotic Activities of Aqueous Extract from Radix Ophiopogon japonicus and Its Two Constituents. Biological \& Pharmaceutical Bulletin, 29, 1267-1270. https://doi.org/10.1248/bpb.29.1267

[15] Kou, J.P., Sun, Y., Lin, Y.W., Cheng, Z.H., Zheng, W., Yu, B.Y. and Xu, Q. (2005) Anti-Inflammatory Activities of Aqueous Extract from Radix Ophiopogon japonicus and Its Two Constituents. Biological \& Pharmaceutical Bulletin, 28, 1234-1238. https://doi.org/10.1248/bpb.28.1234

[16] Xiong, S.L., Li, A.L., Huang, N., Lu, F. and Hou, D.B. (2011) Antioxidant and Immunoregulatory Activity of Different Polysaccharide Fractions from Tuber of Ophiopogon japonicus. Carbohydrate Polymers, 86, 1273-1280. https://doi.org/10.1016/j.carbpol.2011.06.025

[17] Ha, K.T., Yoon, S.J., Choi, D.Y., Kim, D.W., Kim, J.K. and Kim, C.H. (2005) Protective Effect of Lycium chinense Fruit on Carbon Tetrachloride-Induced Hepatotoxicity. Journal of Ethnopharmacology, 96, 529-535. https://doi.org/10.1016/j.jep.2004.09.054

[18] Luo, Q., Cai, Y., Yan, J., Sun, M. and Corke, H. (2004) Hypoglycemic and Hypolipidemic Effects and Antioxidant Activity of Fruit Extracts from Lycium Barbarum. Life Sciences, 76, 137-149. https://doi.org/10.1016/j.lfs.2004.04.056

[19] Chang, R.C.C. and So, K.F. (2008) Use of Anti-Aging Herbal Medicine, Lycium barbarum, against Aging-Associated Diseases. What Do We Know So Far? Cellular and Molecular Neurobiology, 28, 643-652.

https://doi.org/10.1007/s10571-007-9181-x

[20] Jeong, S.C., Kim, S.M., Jeong, Y.T. and Song, C.H. (2013) Hepatoprotective Effect of Water Extract from Chrysanthemum indicum L. Flower. Chinese Medicine, 8, 7. https://doi.org/10.1186/1749-8546-8-7

[21] Hatano, T., Uebayashi, H., Ito, H., Shiota, S., Tsuchiya, T. and Yoshida, T. (1999) Phenolic Constituents of Cassia Seeds and Antibacterial Effect of Some Naphthalenes and Anthraquinones on Methicillin-Resistant Staphylococcus aureus. Chemical \& Pharmaceutical Bulletin, 47, 1121-1127. https://doi.org/10.1248/cpb.47.1121

[22] Shunying, Z., Yang, Y., Huaidong, Y., Yue, Y. and Guolin, Z. (2005) Chemical Composition and Antimicrobial Activity of the Essential Oils of Chrysanthemum indicum. Journal of Ethnopharmacology, 96, 151-158. 
https://doi.org/10.1016/j.jep.2004.08.031

[23] Wei, W., Wu, X.M. and Li, Y.J. (2010) Experimental Methodology of Pharmacology. 4th Edition, People's Medical Publishing Ltd., Beijing.

[24] Pal, R., Vaiphei, K., Sikander, A., Singh, K. and Rana, S.V. (2006) Effect of Garlic on Isoniazid and Rifampicin-Induced Hepatic Injury in Rats. World Journal of Gastroenterology, 12, 636-639. https://doi.org/10.3748/wjg.v12.i4.636

[25] Dong, Y., Huang, J., Lin, X., Zhang, S., Jiao, Y., Liang, T., et al. (2014) Hepatoprotective Effects of Yulangsan Polysaccharide against Isoniazid and Rifampicin-Induced Liver Injury in Mice. Journal of Ethnopharmacology, 152, 201-206. https://doi.org/10.1016/j.jep.2014.01.001

[26] Chen, X., Xu, J., Zhang, C., Yu, T., Wang, H., Zhao, M., et al. (2011) The Protective Effects of Ursodeoxycholic Acid on Isoniazid plus Rifampicin Induced Liver Injury in Mice. European Journal of Pharmacology, 659, 53-60. https://doi.org/10.1016/j.ejphar.2011.03.007

[27] Prabakan, M., Anandan, R. and Devaki, T. (2000) Protective Effect of Hemidesmus indicus against Rifampicin and Isoniazid-Induced Hepatotoxicity in Rats. Fitoterapia, 71, 55-59. https://doi.org/10.1016/S0367-326X(99)00120-3

[28] Steele, M.A., Burk, R.F. and DesPrez, R.M. (1991) Toxic Hepatitis with Isoniazid and Rifampin. A Meta-Analysis. Chest, 99, 465-471. https://doi.org/10.1378/chest.99.2.465

[29] Frydenberg, A.R. and Graham, S.M. (2009) Toxicity of First-Line Drugs for Treatment of Tuberculosis in Children. Tropical Medicine \& International Health, 14, 1329-1337. https://doi.org/10.1111/j.1365-3156.2009.02375.x

[30] Chen, Y., Adhami, N. and Martins-Green, M. (2018) Biological Markers of Harm can Be Detected in Mice Exposed for Two Months to Low Doses of Third Hand Smoke under Conditions that Mimic Human Exposure. Food and Chemical Toxicology, 122, 95-103. https://doi.org/10.1016/j.fct.2018.09.048

[31] Adhami, N., Chen, Y. and Martins-Green, M. (2017) Biomarkers of Disease Can Be Detected in Mice as Early as 4 Weeks after Initiation of Exposure to Third-Hand Smoke Levels Equivalent to those Found in Homes of Smokers. Clinical Science, 131, 2409-2426. https://doi.org/10.1042/CS20171053

[32] Hekimi, S., Lapointe, J. and Wen, Y. (2011) Taking a "Good” Look at Free Radicals in the Aging Process. Trends in Cell Biology, 21, 569-576. https://doi.org/10.1016/j.tcb.2011.06.008

[33] Ayala, A., Munoz, M.F. and Arguelles, S. (2014) Lipid Peroxidation: Production, Metabolism, and Signaling Mechanisms of Malondialdehyde and 4-Hydroxy-2-Nonenal. Oxidative Medicine and Cellular Longevity, 2014, Article ID: 360438. https://doi.org/10.1155/2014/360438

[34] Sarich, T.C., Youssefi, M., Zhou, T., Adams, S.P., Wall, R.A. and Wright, J.M. (1996) Role of Hydrazine in the Mechanism of Isoniazid Hepatotoxicity in Rabbits. Archives of Toxicology, 70, 835-840. https://doi.org/10.1007/s002040050347

[35] Shih, T.Y., Pai, C.Y., Yang, P., Chang, W.L., Wang, N.C. and Hu, O.Y.P. (2013) A Novel Mechanism Underlies the Hepatotoxicity of Pyrazinamide. Antimicrobial Agents and Chemotherapy, 57, 1685-1690. https://doi.org/10.1128/AAC.01866-12

[36] Chowdhury, A., Santra, A., Bhattacharjee, K., Ghatak, S., Saha, D.R. and Dhali, G.K. (2006) Mitochondrial Oxidative Stress and Permeability Transition in Isoniazid and Rifampicin Induced Liver Injury in Mice. Journal of Hepatology, 45, 117-126. https://doi.org/10.1016/j.jhep.2006.01.027 
[37] Santhosh, S., Sini, T.K., Anandan, R. and Mathew, P.T. (2007) Hepatoprotective Activity of Chitosan against Isoniazid and Rifampicin-Induced Toxicity in Experimental Rats. European Journal of Pharmacology, 572, 69-73.

https://doi.org/10.1016/j.ejphar.2007.05.059

[38] Attri, S., Rana, S.V., Vaiphei, K., Sodhi, C.P., Katyal, R., Goel, R.C., et al. (2000) Isoniazid- and Rifampicin-Induced Oxidative Hepatic Injury-Protection by N-Acetylcysteine. Human \& Experimental Toxicology, 19, 517-522.

https://doi.org/10.1191/096032700674230830 